\title{
Monocyte chemoattractant protein-1 (MCP-1) deficiency enhances alternatively activated $M 2$ macrophages and ameliorates insulin resistance and fatty liver in lipoatrophic diabetic A-ZIP transgenic mice
}

\author{
Y. Nio • T. Yamauchi • M. Iwabu • M. Okada-Iwabu • \\ M. Funata $\cdot$ M. Yamaguchi $\cdot$ K. Ueki $\cdot$ T. Kadowaki
}

Received: 7 May 2012 / Accepted: 24 July 2012 / Published online: 16 September 2012

(C) Springer-Verlag 2012

\begin{abstract}
Aims/hypothesis Monocyte chemoattractant protein-1 (MCP-1)/chemokine (C-C motif) ligand (CCL) 2 (CCL2) secreted from white adipose tissue (WAT) in obesity has been reported to contribute to tissue macrophage accumulation and insulin resistance by inducing a chronic inflammatory state. MCP-1 has been shown to be elevated in the fatty liver of lipoatrophic A-ZIP-transgenic (A-ZIP-Tg) mice. Treatment of these mice with the $\mathrm{CC}$ chemokine receptor (CCR) 2 antagonist has been shown to ameliorate the hyperglycaemia, hyperinsulinaemia and hepatomegaly, in conjunction with reducing liver inflammation. However, since CCR2 antagonists can block not only MCP-1 but also MCP-2 (CCL8) and MCP-3 (CCL7), it remains unclear whether MCP-1 secreted from the liver could contribute
\end{abstract}

Electronic supplementary material The online version of this article (doi:10.1007/s00125-012-2710-2) contains peer-reviewed but unedited supplementary material, which is available to authorised users.

Y. Nio • T. Yamauchi $(\bowtie) \cdot$ M. Iwabu $\cdot$ M. Okada-Iwabu • M. Funata $\cdot$ M. Yamaguchi $\cdot$ K. Ueki $\cdot$ T. Kadowaki $(\bowtie)$

Department of Diabetes and Metabolic Diseases,

Graduate School of Medicine, University of Tokyo,

7-3-1 Hongo, Bunkyo-ku,

Tokyo 113-0033, Japan

e-mail: tyamau-tky@umin.net

e-mail:kadowaki-3im@h.u-tokyo.ac.jp

\section{Iwabu}

Department of Integrated Molecular Science on Metabolic Diseases, 22nd Century Medical and Research Center, Graduate School of Medicine, University of Tokyo,

Tokyo, Japan

\section{Okada-Iwabu}

Department of Molecular Medicinal Sciences on Metabolic

Regulation, 22nd Century Medical and Research Center,

Graduate School of Medicine, University of Tokyo,

Tokyo, Japan to hyperglycaemia, hyperinsulinaemia and hepatomegaly in conjunction with liver inflammation, as well as to the M1 and M2 states of macrophage polarisation.

Methods To address these issues, we analysed the effects of targeted disruption of MCP-1 in A-ZIP-Tg mice.

Results MCP-1 deficiency alone or per se resulted in a significant amelioration of insulin resistance in A-ZIP-Tg mice, which was associated with a suppression of extracellular signal-regulated protein kinase (ERK)-1/2 and p38 mitogen-activated protein kinase (p38MAPK) phosphorylation in liver. Although MCP-1 deficiency did not reduce the expression of macrophage markers, it increased the expression of the genes encoding M2 macrophage markers such as Argl and Chi3l3, as well as significantly reducing the triacylglycerol content of livers from A-ZIP-Tg mice.

Conclusions/ interpretation Our data clearly indicated that MCP-1 deficiency improved insulin resistance and hepatic steatosis in A-ZIP-Tg mice and was associated with switching macrophage polarisation and suppressing ERK-1/2 and p38MAPK phosphorylation.

Keywords A-ZIP-Tg mice $\cdot$ Extracellular signal-regulated protein kinase $\cdot$ Hepatic steatosis $\cdot$ Insulin signalling Lipoatrophic diabetes $\cdot$ Macrophage polarisation $\cdot$ Monocyte chemoattractant protein-1

$\begin{array}{ll}\begin{array}{l}\text { Abbreviations } \\ \text { p38MAPK }\end{array} & \text { p38 Mitogen-activated protein kinase } \\ \text { ATMs } & \text { Adipose tissue macrophages } \\ \text { Arg1 } & \text { Arginase-1 } \\ \text { A-ZIP-Tg } & \text { A-ZIP-transgenic } \\ \text { A-ZIP-Tg } \times \mathrm{MCP1}^{-/-} & \text {A-ZIP transgenic MCP-1 } \\ & \begin{array}{l}\text { homozygous knockout } \\ \text { BAT }\end{array} \\ \text { Chi313 } & \text { Chown adipose tissue } \\ & \text { Chitinase 3-like 3 }\end{array}$




$\begin{array}{ll}\text { CCL } & \text { Chemokine (C-C motif) ligand } \\ \text { CCR } & \text { Chemokine (C-C motif) receptor } \\ \text { ERK } & \text { Extracellular signal-regulated } \\ & \text { protein kinase } \\ \text { GTT } & \text { Glucose tolerance test } \\ \text { IR- } \beta & \text { Insulin receptor beta } \\ \text { MCP } & \text { Monocyte chemoattractant protein } \\ \text { SKM } & \text { Skeletal muscles } \\ \text { TG } & \text { Triglycerol } \\ \text { WAT } & \text { White adipose tissue } \\ \text { WT } & \text { Wild-type }\end{array}$

\section{Introduction}

Obesity is associated with increased infiltration of macrophages into the adipose tissues. These adipose tissue macrophages (ATMs) are currently considered to be a major cause of obesity-associated chronic low-grade inflammation via the secretion of a wide variety of inflammatory molecules $[1,2]$, including TNF- $\alpha$, IL-6 [3] and monocyte chemoattractant protein-1 (MCP-1). These inflammatory molecules may have local effects on white adipose tissue (WAT) physiology as well as potential systemic effects on other organs that culminate in insulin resistance.

Among the inflammatory molecules upregulated in the adipose tissues of obese animals and humans, MCP-1 is a member of the cysteine-cysteine (C-C) chemokine family and promotes the migration of inflammatory cells by chemotaxis and integrin activation [4]. Both $M c p-1$ (also known as $\mathrm{Ccl}$ ) mRNA expression in WAT and plasma MCP-1 levels have been found to correlate positively with the degree of obesity in the individual [5]. In addition, increased production of MCP-1 in WAT precedes the production of other macrophage markers during the development of obesity [6]. Mice overproducing MCP-1 in adipocytes showed macrophage recruitment in WAT and exhibited insulin resistance in the skeletal muscles (SKM) and liver [7]. Regarding the mechanisms, it was reported that MCP-1 stimulates the phosphorylation of extracellular signal-regulated protein kinase (ERK) through the C-C chemokine receptor (CCR) 2 [8] and activation of ERK-1/2 induces insulin resistance via decreased tyrosine phosphorylation of insulin receptor beta (IR- $\beta$ ) [7] as well as increased serine phosphorylation of IRS-1 [9].

Macrophage activation has been operationally defined across two separate polarisation states: M1 and M2. M1, or 'classically activated', macrophages are induced by proinflammatory mediators such as lipopolysaccharide, whereas M2, or 'alternatively activated', macrophages generate high levels of anti-inflammatory cytokines such as IL10, arginase-1(Arg1), chitinase 3-like 3 (Chi313) and TGF- $\beta$
[10]. It was previously reported that disruption of MCP-1, or its receptor CCR2, in obese mice resulted in decreased macrophage infiltration in WAT and improved metabolic function $[11,12]$. Moreover, ATMs from obese Ccr2-deficient mice produce M2 markers at levels similar to those seen in lean mice [12]. These data suggest that the MCP-1/ CCR2 axis contributes to macrophage polarisation. Therefore, the phenotypic switch in ATM polarisation is thought to lead to amelioration of insulin resistance.

In contrast to obesity, lipoatrophic diabetes is caused by a deficiency of WAT and is characterised by severe hepatic steatosis and insulin resistance. Moitra and colleagues have generated lipoatrophic A-ZIP transgenic (A-ZIP-Tg) mice, which are profoundly insulin resistant and hyperlipidaemic [13-15]. In addition, these mice exhibit severe hepatic steatosis and at the same time a chronic state of inflammation as indicated by high systemic levels of inflammatory cytokines such as IL-1 $\beta$, IL-6, IL-12 and MCP-1 [16-18]. At the very least, MCP-1 is most abundantly expressed in the liver from A-ZIP-Tg mice [18]. Moreover, treatment of the lipoatrophic A-ZIP-Tg mice with a CCR2 antagonist has been shown to ameliorate the hyperglycaemia, hyperinsulinaemia and hepatomegaly, in conjunction with reducing liver inflammation. However, since CCR2 antagonist can block not only MCP-1, but also MCP-2 (chemokine [C-C motif] ligand [CCL] 8) and MCP-3 (also known as CCL7), it remains unclear whether MCP-1 secreted from liver could contribute to hyperglycaemia, hyperinsulinaemia and hepatomegaly in conjunction with liver inflammation, as well as to $\mathrm{M} 1 / \mathrm{M} 2$ polarisation.

In this study, we hypothesised that MCP-1 secreted from the liver might also play an important role in the regulation of macrophage polarisation and insulin resistance-causing kinases such as ERK and p38 mitogen-activated protein kinase (p38MAPK) in liver. To address these issues, we analysed the effects of a targeted disruption of MCP-1 in lipoatrophic A-ZIP-Tg mice. We showed for the first time that a targeted disruption of MCP-1 alone in lipoatrophic diabetic A-ZIP-Tg mice resulted in decreased ERK-1/2 and p38MAPK phosphorylation and increased alternative M2 activation of macrophages, with at the same time an amelioration of insulin resistance and hepatic steatosis.

\section{Methods}

Generation of A-ZIP-Tg $\times \mathrm{Mcp}^{-/}$mice A-ZIP/F-1 (A-ZIP$\mathrm{Tg}$ ) mice were generous gifts from $\mathrm{C}$. Vinson of the National Cancer Institute at Frederick, MD, USA. $\mathrm{Mcpl}^{-/-}$mice were purchased from the Jackson Laboratory (Bar Harbor, ME, USA) and C57BL/6 mice from CLEA Japan (Fujinomiya, Shizuoka, Japan). A-ZIP/F-1 mice were on an FVB/N (FVB) background. MCP-1 homozygous-knockout mice 
were on a C57BL/6 background, which had been backcrossed to C57BL/6 mice for 10 generations.

To generate A-ZIP-Tg $\mathrm{McpI}^{+/}$mice, conceptuses that were obtained by in vitro fertilisation of ova from $\mathrm{Mcpl}^{-/}$ mice and sperm from A-ZIP-Tg mice were implanted into pseudo-pregnant foster mothers as previously described [19]. To generate A-ZIP-Tg $\times$ MCP-1 knockout $\left(\mathrm{Mcpl}^{-/-}\right)$mice and $\mathrm{McPI}^{-/-}$mice, conceptuses that had been obtained by in vitro fertilisation of ova from MCP-1 homozygous-knockout mice and sperm from A-ZIP-Tg $\times$ MCP-1 heterozygous-knockout mice were implanted into pseudo-pregnant foster mothers. To generate wild-type (WT) mice and A-ZIP-Tg mice, conceptuses that had been obtained by in vitro fertilisation of ova from C57BL/6 mice and sperm from A-ZIP-Tg $\times$ MCP-1 heterozygous-knockout mice were implanted into pseudopregnant foster mothers. All experiments in this study were conducted on female mice.

In this study, we generated WT mice, $\mathrm{Mcpl}^{-/-}$mice, A-ZIP-Tg mice and A-ZIP-Tg $\times \mathrm{Mcp}^{-/-}$mice which were on an FVB/B6 F2 background. This breeding strategy was used to improve the viability of the offspring as AZIP-Tg mice on an FVB background have poor survival [13]. As there was no difference in body weight, plasma glucose levels in the fed state and liver weight between WT mice and $\mathrm{Mcpl}^{-/}$mice (see electronic supplementary material [ESM] Fig. 1), and previous reports had also shown that the body weight, adipose weight, insulin
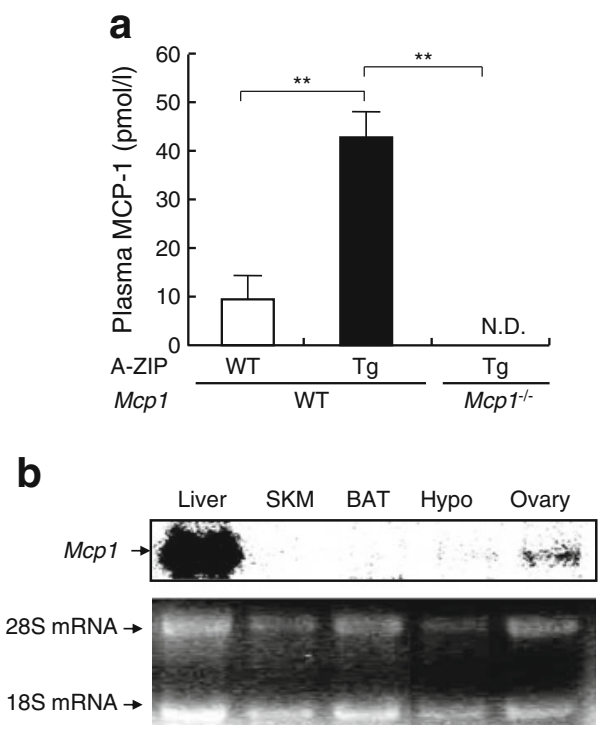

Fig. 1 Plasma MCP-1 concentration and tissue distribution of Mcp 1 mRNA in A-ZIP-Tg mice. (a) Plasma concentrations of MCP-1 were measured in WT (white bars), A-ZIP-Tg (black bars) and A-ZIP$\mathrm{Tg} \times \mathrm{Mcpl}^{-/-}$(grey bars) 15-week-old mice in the fed state. Data are means \pm SEM. WT mice, $n=5$; A-ZIP-Tg mice, $n=17$; A-ZIP$\mathrm{Tg} \times \mathrm{McpI}^{-/}$mice, $n=16 .{ }^{* *} p<0.01$ vs A-ZIP-Tg mice. ND, not detected. (b) Northern blot analysis of $M c p 1$ mRNA expressed in liver, SKM, BAT, Hypo (hypothalamus) and ovary of 15 -week-old A-ZIP-Tg mice tolerance and glucose tolerance of $\mathrm{Mcpl}^{-/}$mice fed normal chow did not differ from those of WT control mice [11], we analysed and compared the WT mice, AZIP-Tg mice and A-ZIP-Tg $\times M c p 1^{-/}$mice. The increase in plasma MCP-1 level of A-ZIP-Tg male mice (ESM Fig. 2a) was similar to that of A-ZIP-Tg female mice (Fig. 1a). MCP-1 deficiency significantly ameliorated hyperglycaemia (ESM Fig. 2b) but not hyperinsulinaemia in A-ZIP-Tg male mice (ESM Fig. 2c). These data suggested that the insulin-sensitive phenotype of A-ZIP-Tg$\mathrm{Mcpl}^{-/}$mice was prominent in female mice compared with male mice. We thus used female mice in this study.

Mice Mice were housed in cages and maintained on a 12$\mathrm{h}$ light/dark cycle. For all experiments, the diet was standard chow (CE-2; CLEA Japan) with the following composition: $25.6 \%(\mathrm{wt} / \mathrm{wt}$ ) protein, $3.8 \%$ fibre, $6.9 \%$ ash, $50.5 \%$ carbohydrates, $4 \%$ fat and $9.2 \%$ water [19-21]. The animal care and use procedures were approved by the Animal Care Committee of the University of Tokyo.

Northern blot analysis Northern blotting was carried out according to the method described previously [20,21]. Total RNA was extracted from various tissues with TRIzol reagent according to the manufacturer's instructions (Invitrogen, Carlsbad, CA, USA). Total RNA $(15 \mu \mathrm{g})$ was loaded onto a $1.3 \%$ agarose gel and transferred to a nylon membrane (Hybond N+; GE Healthcare Life Sciences, Hino, Tokyo, Japan). MCP-1 coding sequence cDNA was used as the probe template. The cDNA probe template of MCP-1 was prepared by RT-PCR using specific primers. The forward primer was $5^{\prime}$-CCATGCAGGTCCCTGTC-3' and the reverse primer was $5^{\prime}$-CTAGTTCACTGTCACAC-3', as previously described [7]. The corresponding bands were quantified by exposure of BAS2000 to the filters and measurement with BAStation software (Fuji Film, Minato-ku, Tokyo, Japan).

Real-time quantitative PCR For real-time quantitative PCR analysis, cDNA synthesised from total RNA was analysed. For quantification of gene expression, a set of predesigned primers and probes for each gene (Assays-on-Demand; Applied Biosystems, Carlsbad, CA, USA) were used. These were mouse MCP-1, Mm00441242 m1; mouse MCP-2: Mm01297183_m1; mouse MCP-3, Mm00443113_m1; mouse CCR 2 , Mm99999051_gH; mouse Emrr1, Mm00802530_m1; mouse CD68, Mm00839636 g1; mouse Chi313, Mm00657889 mH; mouse TGF- $\beta$, Mm03024053_m1; mouse Arg1, Mm01190441_g1; mouse PPARa: Mm00440949 m1; mouse Acyl-coA oxidase: Mm00443579_m1; mouse UCP2: Mm00495907_g1; mouse SREBP1c: Mm00550338 m1 and mouse SCD1: Mm00772290_m1. 
The primer sets and the probe for mouse cyclophilin were as follows: the forward primer was 5'-GGTCCT GGCATCTTGTCCAT-3', the reverse primer was 5'CAGTCTTGGCAGTGCAGATAAAA-3'; and the probe was 5'-CTGGACCAAACACAAACGGTTCCCA-3'. The relative amount of each transcript was normalised to the amount of mouse cyclophilin mRNA.

Blood sample assays and in vivo glucose homeostasis Glucose tolerance tests (GTTs) were conducted as previously described with slight modifications [20, 21]. For the GTTs, mice were deprived of food for $6 \mathrm{~h}$ and then orally administered with D-glucose ( $1.5 \mathrm{~g}$ per $\mathrm{kg}$ body weight). Plasma glucose and plasma triglycerol (TG) levels in the fed state were determined using a glucose B-test and TG E-type test (Wako Pure Chemical Industries, Yodogawa-ku, Osaka, Japan), respectively. Plasma insulin levels were measured with an insulin immunoassay (Shibayagi, Shibukawa, Gunma, Japan). Plasma MCP-1 levels in the fed state were measured using a mouse immunoassay kit (Pierce Biotechnology, Rockford, IL, USA).

Antibodies Mouse monoclonal anti-phosphotyrosine antibody 4G10 ( $\alpha \mathrm{PY})$ was purchased from Merck Millipore (Billerica, MA, USA). Rabbit polyclonal antibody to IR- $\beta$ was purchased from Santa Cruz Biotechnology (Santa Cruz, CA, USA). Rabbit polyclonal antibodies against ERK-1/2, phospho-ERK-1/2 (Thr202/Tyr204), p38MAPK, phosphor-p38MAPK (Thr180/ Tyr182), Akt and phospho-Akt (Ser-473) were purchased from Cell Signaling Technology (Danvers, MA, USA).

Immunoblotting and immunoprecipitation Immunoblotting and immunoprecipitation were conducted as previously described [22]. In brief, in the fed state, the skeletal muscles from the hind limbs or livers were removed. The samples were homogenised in ice-cold 1\% Nonidet P-40-buffer $(25 \mathrm{mmol} / \mathrm{l}$ Tris- $\mathrm{HCl}$ [pH 7.4], $10 \mathrm{mmol} / 1$ sodium orthovanadate, 10 $\mathrm{mmol} / \mathrm{l}$ EGTA and $1 \mathrm{mmol} / \mathrm{l}$ phenylmethylsulfonyl fluoride) and centrifuged. For immunoblotting, muscle homogenates containing $5 \mathrm{mg}$ of total protein or liver homogenates containing $15 \mathrm{mg}$ of total protein were incubated with the indicated primary antibodies and horseradish peroxidase-conjugated anti-mouse-IgG secondary antibody and were detected with enhanced chemiluminescence (ECL) reagent (GE Healthcare Life Sciences). For immunoprecipitation, muscle homogenates containing $5 \mathrm{mg}$ of total protein or liver homogenates containing $15 \mathrm{mg}$ of total protein were also incubated with the indicated antibodies followed by addition of protein GSepharose. The immunoprecipitates were washed with $1 \%$ Nonidet P-40-buffer three times. The immunoprecipitates were subjected to immunoblotting with the indicated primary antibodies and horseradish peroxidase-conjugated anti-mouse-IgG secondary antibody and were detected with ECL reagent.
Histological analyses and TG content in liver Livers from WT mice, A-ZIP-Tg mice and A-ZIP-Tg $\times \mathrm{Mcpl}^{-/-}$mice were fixed overnight in $10 \%$ formalin (vol./vol.). Samples were routinely embedded in paraffin. Approximately $5 \mu \mathrm{m}$ thick slices obtained from these liver samples were stained with haematoxylin and eosin. The liver homogenates were extracted and their TG content was determined as previously described [19].

Statistical analysis Results are expressed as means \pm SEM. The Student's $t$ test was performed to compare two groups. Data involving more than two groups were assessed by ANOVA. Values of $p<0.05$ were considered statistically significant.

For details of the hyperinsulinaemic-euglycaemic clamp study and antibodies used in immunoprecipitation and immunoblotting, please refer to the ESM Methods.

\section{Results}

Plasma MCP-1 concentration and Mcp-1 mRNA expression in fatty liver were increased in lipoatrophic A-ZIP-Tg mice Many previous studies have reported elevated plasma MCP-1 concentration and Mcp-1 mRNA expression in WAT of obese and diabetic mice [7, 11, 23-25]. As reported [18], plasma MCP-1 concentrations were significantly elevated in A-ZIPTg mice compared with WT mice (Fig. 1a). A previous study showed that, in WT mice, $M c p-1$ mRNA was not detected in any tissues [11], whereas in A-ZIP-Tg mice, as previously reported [18], Mcp-1 mRNA was most abundantly expressed in the liver among all the tissues we examined, including brown adipose tissue (BAT), except for WAT, because lipoatrophic A-ZIP-Tg mice still have BAT but not WAT, as reported in other studies [12-14, 18] (Fig. 1b). It has been reported that MCP-1 is abundantly expressed in Kupffer cells, as well as in hepatocytes in the liver [26,27]

MCP-1 deficiency decreased body weight gain and liver weight in A-ZIP-Tg mice To clarify the pathophysiological roles of elevated MCP-1 levels in lipoatrophic A-ZIP-Tg mice, we generated A-ZIP-Tg $\times \mathrm{Mcpl}^{-/}$mice. MCP-1 deficiency in A-ZIP-Tg mice resulted in decreased body weight compared with A-ZIP-Tg mice (Fig. 2a), although the food intake of A$\mathrm{ZIP}-\mathrm{Tg} \times \mathrm{Mcp}^{-/}$mice was not significantly different from that seen in A-ZIP-Tg mice (ESM Table 1). Furthermore, the liver weight of A-ZIP-Tg $\times \mathrm{Mcpl}^{-/}$mice was decreased compared with that of A-ZIP-Tg mice (Fig. 2b).

MCP-1 deficiency ameliorated glucose tolerance in lipoatrophic A-ZIP-Tg mice A-ZIP-Tg $\times M c p 1^{-/-}$mice showed amelioration of hyperglycaemia, hyperinsulinaemia and hypertriacylglycerolaemia compared with A-ZIP-Tg mice 
a
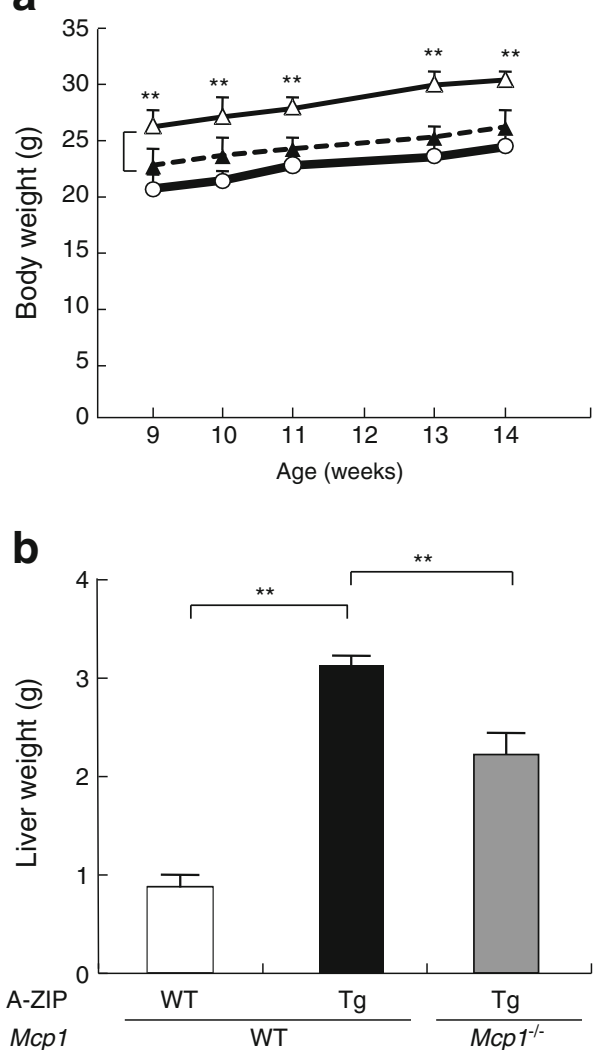

Fig. 2 Body weight and liver weight of WT mice, A-ZIP-Tg mice and A-ZIP-Tg $\times \mathrm{Mcpl}^{-/-}$mice. (a) Body weight of WT mice (white circles), A-ZIP-Tg mice (white triangles) and A-ZIP-Tg $\times \mathrm{Mcpl}^{-/-}$mice (black triangles) were measured from 9 to 14 weeks of age, as indicated. (b) Liver weight of WT (white bars), A-ZIP-Tg (black bars) and A-ZIP$\mathrm{Tg} \times \mathrm{Mcpl}^{-1-}$ (grey bars) in 15-week-old mice. Data are means $\pm \mathrm{SEM}$. WT mice, $n=5$; A-ZIP-Tg mice, $n=17$; A-ZIP-Tg $\times M c p 1^{-/}$mice, $n=$ 16. ${ }^{* *} p<0.01$ vs A-ZIP-Tg mice

(Fig. 3a-c). To further clarify the effects of MCP-1 deficiency in A-ZIP-Tg mice on glucose tolerance and insulin sensitivity, we performed GTTs. Plasma glucose levels and plasma insulin levels during the GTTs were significantly lower in A-ZIP-Tg $\times \mathrm{Mcpl}^{-/}$mice than in A-ZIP-Tg mice (Fig. 3d,e), suggesting that MCP-1 deficiency in A-ZIP-Tg mice partially ameliorated glucose intolerance. In WT mice, plasma insulin levels were increased after glucose administration, whereas they were remarkably decreased in A-ZIP$\mathrm{Tg}$ and A-ZIP-Tg- $\mathrm{Mcpl}^{-/-}$mice, consistent with previous observations that prolonged hyperinsulinaemia due to severe insulin resistance can result in decreased glucosestimulated insulin-secretion, in particular decreased earlyphase insulin secretion [28]. A hyperinsulinaemiceuglycaemic clamp study revealed that the glucose infusion rate was significantly increased in A-ZIP-Tg- $M c p 1^{-/}$mice compared with A-ZIP-Tg mice (ESM Fig. 3), indicating that disruption of MCP-1 in A-ZIP-Tg mice could ameliorate insulin resistance.
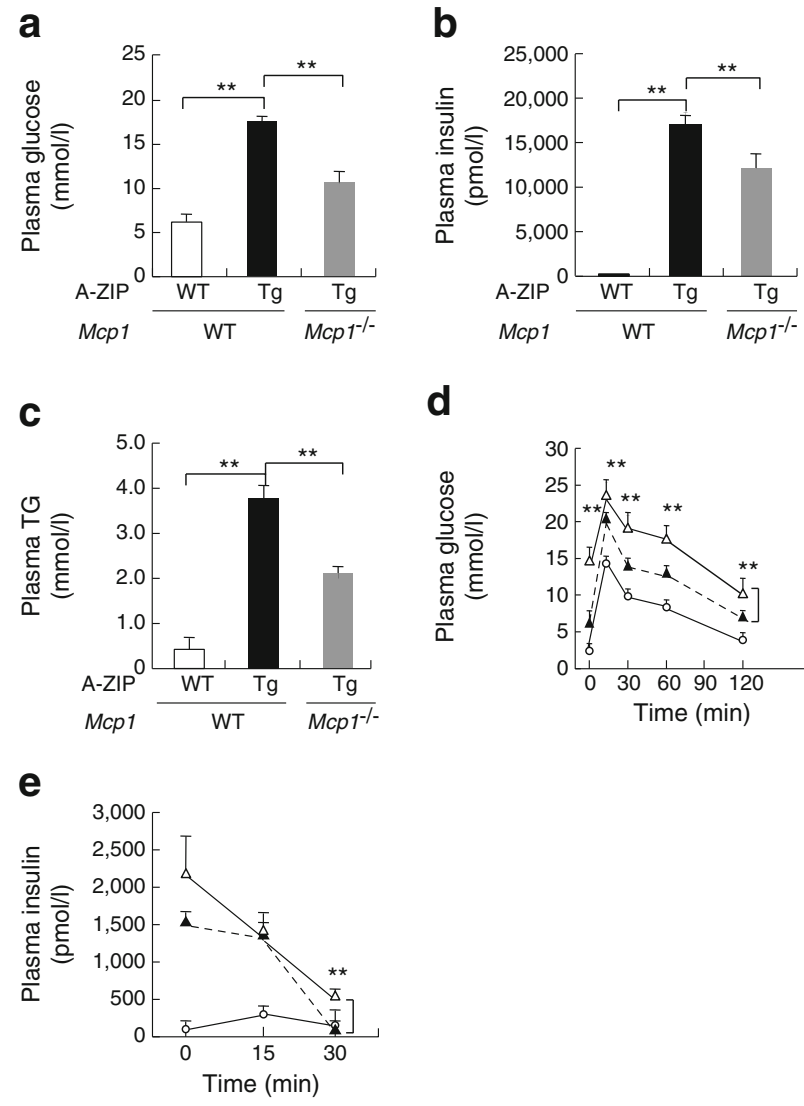

d

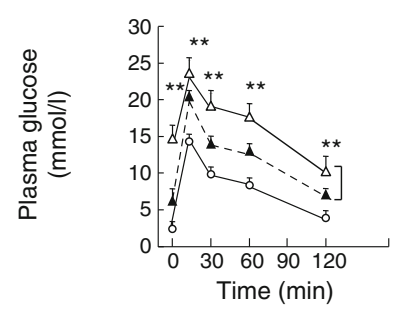

Fig. 3 MCP-1 deficiency in A-ZIP-Tg mice ameliorates glucose intolerance and insulin sensitivity. Plasma values for glucose (a), insulin (b) and TG (c) were measured in 15-week-old WT (white bars), A-ZIPTg (black bars) and A-ZIP-Tg $\times M c p 1^{-/}$(grey bars) mice. Plasma glucose (d) and plasma insulin (e) during GTT (1.5 g glucose per kilogram body weight) in WT (white circles), A-ZIP-Tg (white triangles) and A-ZIP-Tg $\times \mathrm{Mcpl}^{-/-}$(black triangles) in 15-week-old mice. Data are means \pm SEM. WT mice, $n=5$; A-ZIP-Tg mice, $n=17$; A-ZIP$\mathrm{Tg} \times \mathrm{Mcpl}^{-/}$mice, $n=16 .{ }^{* *} p<0.01$ vs A-ZIP-Tg mice

Livers from A-ZIP-Tg $\times \mathrm{McPl}^{-/}$mice showed increased markers of alternatively activated M2 macrophages Many studies have demonstrated that macrophage infiltration and levels of proinflammatory cytokines such as MCP-1 and TNF- $\alpha$ are increased in WAT of human obese individuals and several models of rodent obesity [29, 30]. We examined whether the expression of macrophage marker genes would be elevated in other tissues than WAT, such as liver, SKM and BAT, in A-ZIP-Tg mice. The mRNA expressions of Mcp-1, Mcp-3 (Ccl7), Ccr2, Emr1 (EGF-like module containing, mucin-like, hormone receptor-like 1) and Cd68 (CD68 antigen) were increased in the liver (Fig. 4a-e), SKM (ESM Fig. 4) and BAT (ESM Fig. 5) of A-ZIP-Tg mice compared with WT mice. The mRNA expressions of $M c p-2$ were significantly increased in the liver and BAT of A-ZIP-Tg mice compared with those of WT mice. MCP-1 deficiency did not significantly decrease the expressions of $M c p-2$ in the liver, SKM and BAT in A-ZIP-Tg mice (ESM Fig. 6). Unexpectedly, MCP-1 
Fig. 4 Effects of MCP-1 deficiency on the expression of macrophage marker genes in liver. $M c p-1$ (a), $M c p-3$ (b), Ccr2 (c), Emr1 (d) and Cd68 (e) mRNA levels and the mRNA ratio of $C h i 3 l 3 / C d 68$ (f), $\operatorname{Arg} 1 / C d 68$ (g) and $T g f-\beta /$ Cd68 (h) in livers from WT (white bars), A-ZIP-Tg (black bars) and A-ZIP-Tg $\times M_{c p 1^{-/}}$ (grey bars) in 15-week-old mice in the fed state were analysed by real-time quantitative PCR. Data are means \pm SEM. WT mice, $n=5$; A-ZIP-Tg mice, $n=$ 17; A-ZIP-Tg $\times \mathrm{Mcpl}^{-/}$mice, $n=16 . * p<0.05$ vs A-ZIP-Tg mice
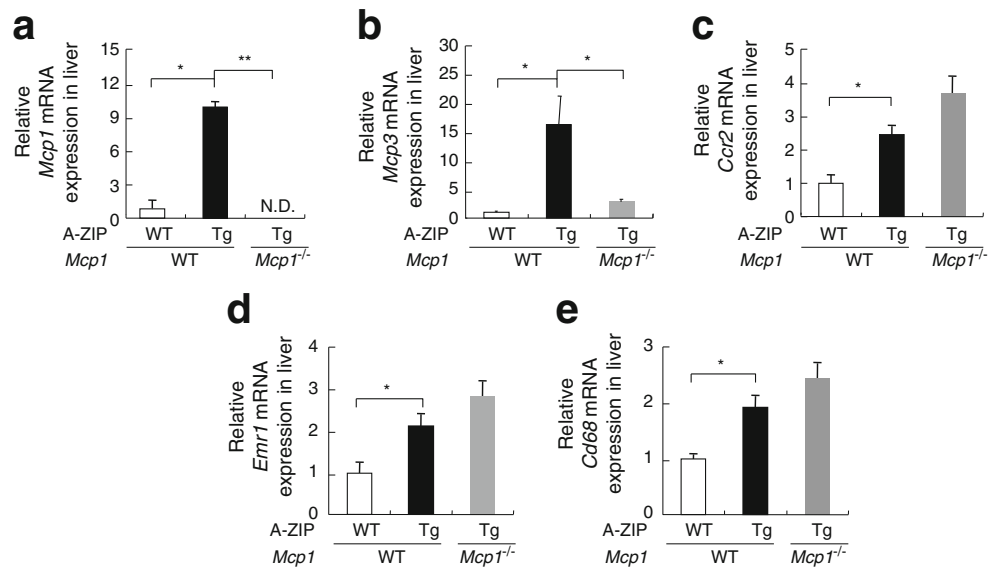

f

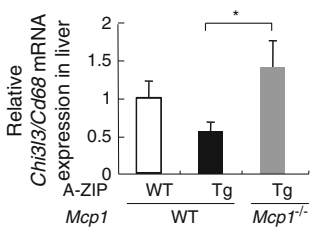

g

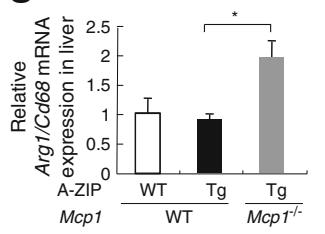

h

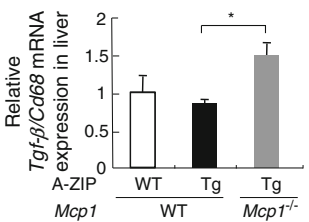

deficiency did not decrease the expression of macrophage marker genes in other tissues than WAT, such as the liver, SKM and BAT in A-ZIP-Tg mice.

Because a previous report showed that ATMs isolated from obese $C$ cr2-deficient mice expressed alternatively activated M2-macrophage markers [12], we hypothesised that livers from A-ZIP-Tg $\times \mathrm{Mcpl}^{-/-}$mice might contain M2-polarised macrophages more abundantly. Interestingly, characteristic M2-macrophage marker genes such as Chi3l3, Argl and $T g f b 1$ were significantly increased in livers from A-ZIP$\mathrm{Tg} \times \mathrm{Mcpl}^{-/}$mice (Fig. $4 \mathrm{f}-\mathrm{h}$ ). In contrast to liver, characteristic M2-macrophage marker genes such as Chi3l3, Argl and $T g f b 1$ were not significantly changed in SKM and BAT from A-ZIP-Tg $\times \mathrm{Mcpl}^{-/-}$mice (ESM Fig. 7). Thus, MCP-1 deficiency did not reduce macrophage markers but rather induced macrophages to shift to a M2-polarised state in livers from AZIP-Tg mice. These data suggest that MCP-1 deficiency enhances M2 polarisation in livers of A-ZIP-Tg mice.
MCP-1 deficiency decreased liver TG content in A-ZIP-Tg mice It was previously reported that A-ZIP-Tg mice showed dyslipidaemia and severe hepatic steatosis [13]. Liver weights were markedly reduced in A-ZIP-Tg $\times \mathrm{Mcpl}^{-/}$mice compared with A-ZIP-Tg mice (Fig. 2b). Representative histological sections of livers showed that MCP-1 deficiency decreased TG accumulation (Fig. 5a). We subsequently determined that the TG content in livers from A-ZIP-Tg $\times \mathrm{Mcpl}^{-/-}$ mice was significantly less than that in livers from A-ZIP-Tg mice (Fig. 5b). To clarify the mechanism by which hepatic steatosis was improved, we studied the expression of genes involved in lipid and energy metabolism and found that expression of Ppara mRNA tended to be increased and expression of genes involved in energy dissipation such as Ucp2 significantly increased in A-ZIP-Tg- $\mathrm{Mcpl}^{-/}$mice compared with A-ZIP-Tg mice, whereas the expression of genes involved in lipogenesis such as Srebp-1c (Srebf1) and Scd-1 was not significantly changed (ESM Fig. 8). a

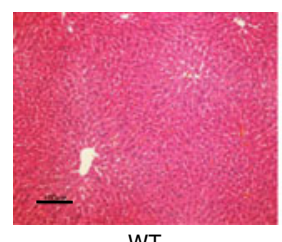

WT

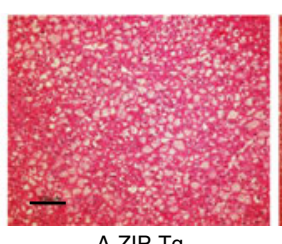

A-ZIP-Tg

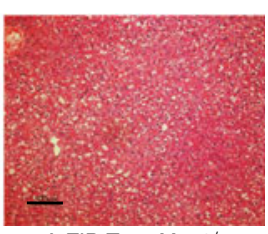

$\mathrm{A}-\mathrm{ZIP}-\mathrm{Tg} \times \mathrm{Mcp}^{-1-}$

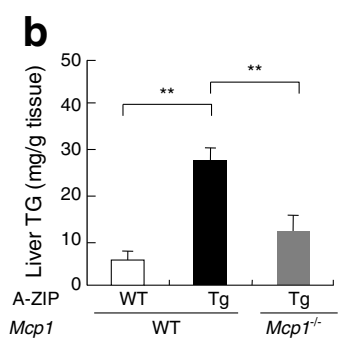

Fig. 5 (a) Histological analysis and TG content of liver. Sections of livers were stained with haematoxylin and eosin (Scale bars, $100 \mu \mathrm{m}$ ). (b) TG content of livers from WT (white bars), A-ZIP-Tg (black bars) and A-ZIP-Tg $\times \mathrm{Mcpl}^{-/}$(grey bars) mice. Data are means \pm SEM. WT mice, $n=5$; A-ZIP-Tg mice, $n=17$; A-ZIP-Tg $\times M c p 1^{-1-}$ mice, $n=16$. $* * p<0.01$ vs A-ZIP-Tg mice 
MCP-1 deficiency enhanced insulin signalling in livers of A-ZIP-Tg mice Previous studies have reported that MCP-1 induces phosphorylation of ERK through activation of CCR2 in SKM, myocytes, kidney and leukaemia cells [7, 8, 31, 32]. Moreover, Bost et al reported that mice lacking ERK-1 were protected from high-fat diet-induced obesity and insulin resistance [33]. Thus, we hypothesised that inhibition of ERK signalling would improve insulin signalling in A-ZIP-Tg mice, so we studied phosphorylation of ERK-1/2 in livers from A-ZIP-Tg mice and A-ZIP$\mathrm{Tg} \times \mathrm{Mcpl}^{-/-}$mice. In fact, ERK-1/2 and p38MAPK phosphorylation were significantly increased in livers from A-ZIP-Tg mice compared with WT mice. Conversely, phosphorylation of ERK-1/2 was decreased in livers from A-ZIP-Tg $\times \mathrm{Mcpl}^{-/}$mice compared with A-ZIP-Tg mice (Fig. 6a). We next examined the effects of MCP-1 deficiency on insulin signalling in livers from A-ZIP-Tg mice. In livers from A-ZIP-Tg $\times \mathrm{Mcpl}^{-/}$mice, tyrosine phosphorylation of IR- $\beta$ and serine phosphorylation of $\mathrm{Akt}$ were significantly increased compared with that seen in A-ZIP-Tg mice (Fig. 7). Moreover, Ser 612 in IRS-1 has
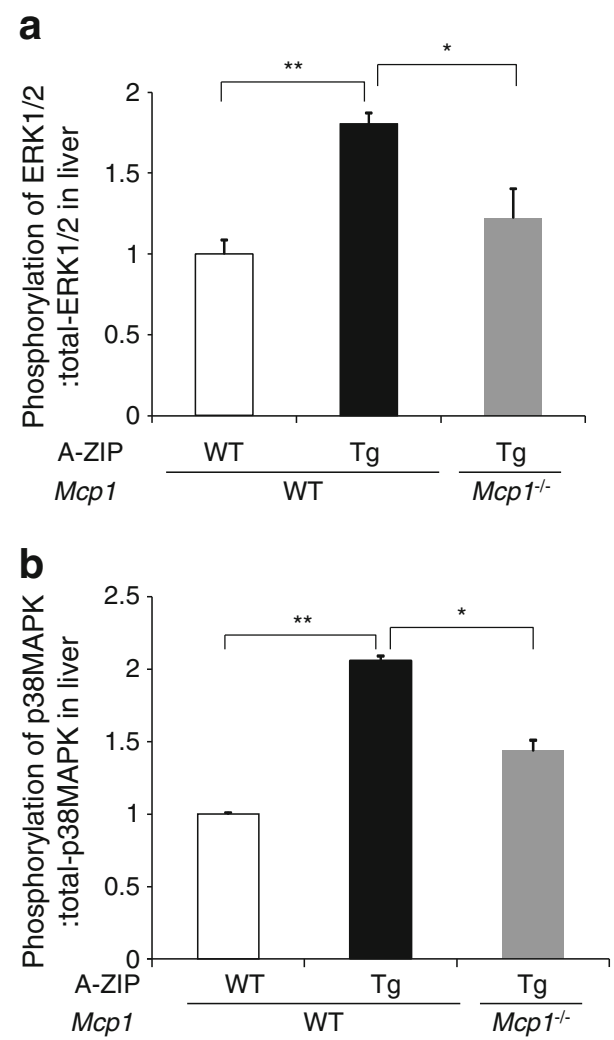

Fig. 6 MCP-1 deficiency in livers from A-ZIP-Tg mice results in the phosphorylation of ERK-1/2 and p38MAPK. Phosphorylation of ERK1/2 (a) and p38MAPK (b) in livers from WT, A-ZIP-Tg and AZIP-Tg $\times \mathrm{Mcpl}^{-/}$15-week-old mice in the fed state were analysed by immunoblotting. The relative amount of each protein was normalised to the total amount of ERK-1/2 or p38MAPK and the ratio is shown. Data are means $\pm \operatorname{SEM}$ ( $n=3$ per group). ${ }^{*} p<0.05,{ }^{*} p<0.01$ vs WT or A-ZIP-Tg mice been reported to be phosphorylated by ERK [33], which would result in inhibition of insulin signalling. The amount of Ser 612 phosphorylation in IRS-1 was significantly decreased in livers from A-ZIP-Tg $\times \mathrm{Mcpl}^{-/-}$mice compared with A-ZIP-Tg mice (ESM Fig. 9). Furthermore, in SKM from A-ZIP-Tg $\times \mathrm{Mcpl}^{-/-}$mice, tyrosine phosphorylation of IR- $\beta$ was significantly, and serine phosphorylation of Akt tended to be, increased compared with A-ZIP-Tg mice (ESM Fig. 10).

\section{Discussion}

MCP-1/CCL2 secreted from WAT in obesity has been reported to contribute to tissue macrophage accumulation and insulin resistance by inducing a chronic inflammatory state. MCP-1 has been shown to be elevated in the fatty liver of lipoatrophic A-ZIP-Tg mice. Treatment of these mice with CCR2 antagonist has been shown to ameliorate their hyperglycaemia, hyperinsulinaemia and hepatomegaly, in conjunction with a reduction in liver inflammation.
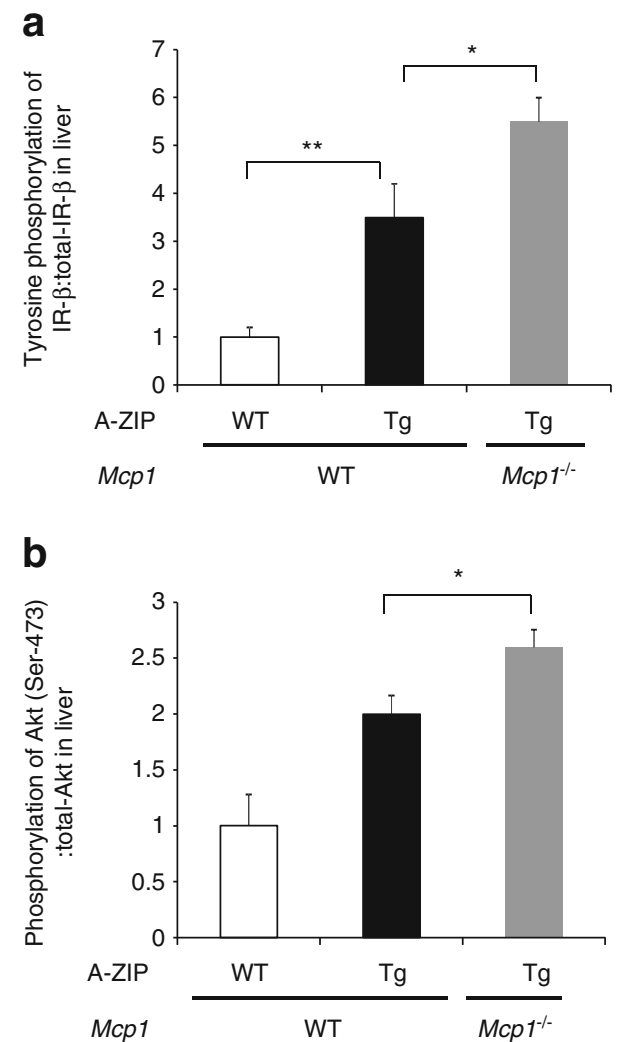

Fig. 7 MCP-1 deficiency in livers from A-ZIP-Tg mice results in increased insulin signalling. Tyrosine phosphorylation of IR- $\beta$ (a) and phosphorylation of Akt (Ser-473) (b) in livers from WT, A-ZIP-Tg and A-ZIP-Tg $\times \mathrm{Mcpl}^{-1} 15$-week-old mice in the fed state were analysed by immunoblotting. The relative amount of each protein was normalised to the total amount of IR- $\beta$ or Akt and the ratio is shown. Data are means \pm SEM ( $n=3$ per group). ${ }^{*} p<0.05,{ }^{* *} p<0.01$ vs WT or A-ZIP-Tg mice 
However, since CCR2 antagonist can block not only MCP1, but also MCP-2 and MCP-3, it remains unclear whether MCP-1 secreted from liver could contribute to hyperglycaemia, hyperinsulinaemia and hepatomegaly in conjunction with liver inflammation, as well as to M1/M2 polarisation.

To address these issues, we analysed the effects of targeted disruption of MCP-1 in A-ZIP-Tg mice. In the current study, we showed for the first time that targeted disruption of MCP-1 alone in lipoatrophic diabetic A-ZIP-Tg mice ameliorated insulin resistance and hepatic steatosis, which was associated with decreased ERK-1/2 and p38MAPK phosphorylation (Fig. 6) and alternative M2 activation of macrophages. Although food intake was not significantly different when adjusted by body weight (ESM Table 1), the A-ZIP-Tg mice with MCP-1 deficiency tended to exhibit increased Ppara and exhibited significantly increased Ucp2 expression in the liver (ESM Fig. 8), raising the possibility that the body weight and liver weight reduction (ESM Table 2) were due to increased energy expenditure, and that it could also contribute to the reduction in liver triacylglycerol content. However, it has been technically extremely difficult to prepare enough A-ZIP-Tg $\times \mathrm{Mcpl}^{-/-}$mice to unequivocally prove increased energy expenditure in these mice. We would like to carry out this experiment in a future study.

The amounts of tyrosine phosphorylation of IR- $\beta$ and serine phosphorylation of Akt were increased in livers from A-ZIP-Tg $\times \mathrm{Mcpl}^{-/-}$mice (Fig. 7). These data suggested that the amelioration of insulin resistance by disruption of MCP1 in A-ZIP-Tg mice, such as increased tyrosine phosphorylation of IR- $\beta$ and serine phosphorylation of Akt in liver, seemed to be related to decreased ERK-1/2 and p38MAPK activation [34], at least in part.

Although the disruption of MCP-1 in A-ZIP-Tg mice improved insulin resistance, the gene expression of macrophage markers such as Emrl and Cd68 in the liver were not decreased compared with those seen in A-ZIP-Tg mice (Fig. $4 d, e)$. It was recently reported that the induction of M2 markers in resident macrophages in the liver controls hepatic lipid metabolism [35]. In this study, the M2-specific genes Chi3l3 and $\mathrm{Argl}$ were increased in livers from AZIP-Tg $\times \mathrm{Mcpl}^{-/}$mice compared with A-ZIP-Tg mice (Fig. 4f,g). These data are consistent with the results that ATMs from obese $C$ cr2-deficient mice express M2 markers at levels similar to those found in lean mice [12]. However, treatment with CCR2 antagonist reduced the macrophage marker gene $C d 68$ mRNA and Tnf- $\alpha$ mRNA in the livers of A-ZIP-Tg mice [18]. These results might be derived from differences between a chronic and an acute inhibition of CCR2 or between targeted disruption of MCP-1 and simultaneous inhibition of MCP-1/MCP-2/MCP-3 by CCR2 antagonist in the liver of A-ZIP-Tg mice.

Previous studies have shown that CCR2 promoted obesityinduced hepatic steatosis in $d b / d b$ mice [36], that a CCR2 antagonist (propagermanium) also reduced liver TG content in $d b / d b$ mice [37], and that another CCR2 antagonist (RS 504393) ameliorated hepatomegaly but did not decrease hepatic TG content significantly $(p=0.144)[18]$. These results raised the possibility that inhibition of MCP-1 and/or MCP-2 and/or MCP-3 by CCR2 inhibition could be involved in the suppression of hepatic steatosis. In this study, we showed for the first time that targeted disruption of MCP-1 in A-ZIP-Tg mice by itself exhibited decreased liver TG content in the liver (Fig. 5b), clearly indicating that MCP-1 plays an important role in the onset of hepatic steatosis in the liver in A-ZIP-Tg mice.

In conclusion, we showed for the first time that targeted disruption of MCP-1 by itself ameliorated insulin resistance and hepatic steatosis, and at the same time decreased phosphorylation of ERK-1/2 and p38 MAPK (increased tyrosine phosphorylation of IR- $\beta$ and serine phosphorylation of Akt) and induced alternative $\mathrm{M} 2$ activation of macrophages in the fatty liver from lipoatrophic diabetic A-ZIP-Tg mice. These results suggest that MCP-1 derived from tissues other than WAT, such as fatty liver, could also play an important role in the regulation of whole body insulin sensitivity, hepatic steatosis, macrophage polarisation and phosphorylation of ERK-1/2 and p38 MAPK. The current study also suggests that modulating MCP-1 in the liver would be useful therapy for diabetes and fatty liver.

Acknowledgements We are grateful to K. Miyata for technical assistance.

Funding This work was supported by Grant-in-aid for Scientific Research (S) (20229008) (to T.K.), Grant-in-aid for Scientific Research on Innovative Areas (Research in a proposed research area) 'Molecular Basis and Disorders of Control of Appetite and Fat Accumulation' (to T.Y.), Funding Program for Next Generation World-Leading Researchers (NEXT Program) (to T.Y.), Targeted Proteins Research Program (to T.K.), the Global COE Research Program (to T.K.) and Translational Systems Biology and Medicine Initiative (to T.K.) from the Ministry of Education, Culture, Sports, Science and Technology of Japan.

Duality of interest The authors declare that there is no duality of interest associated with this manuscript.

Contribution statement YN researched, analysed and interpreted data and wrote the manuscript. TY wrote the manuscript, researched, analysed and interpreted data and contributed to the conception and design of this study. MI and MO-I researched, analysed and interpreted data and edited the manuscript. MF and MY researched data and edited the manuscript. KU analysed data and edited the manuscript. TK contributed to the conception and design of this study and edited the manuscript. YN, TY and TK are the guarantors for the content of this article. All authors have approved the final version of the manuscript.

\section{References}

1. Wellen KE, Hotamisligil GS (2005) Inflammation, stress, and diabetes. J Clin Invest 115:1111-1119 
2. Kershaw EE, Flier JS (2004) Adipose tissue as an endocrine organ. J Clin Endocrinol Metab 89:2548-2556

3. Arranz J, Soriano A, Garcia I et al (2003) Effect of proinflammatory cytokines (IL-6, TNF-alpha, IL-1beta) on hemodynamic performance during orthotopic liver transplantation. Transplant Proc 35:1884-1887

4. Boring L, Gosling J, Cleary M, Charo IF (1998) Decreased lesion formation in $\mathrm{CCR} 2^{-1-}$ mice reveals a role for chemokines in the initiation of atherosclerosis. Nature 394:894-897

5. Weisberg SP, McCann D, Desai M, Rosenbaum M, Leibel RL, Ferrante AW Jr (2003) Obesity is associated with macrophage accumulation in adipose tissue. J Clin Invest 112:1796-1808

6. Xu H, Barnes GT, Yang Q et al (2003) Chronic inflammation in fat plays a crucial role in the development of obesity-related insulin resistance. J Clin Invest 112:1821-1830

7. Kamei N, Tobe K, Suzuki R et al (2006) Overexpression of monocyte chemoattractant protein-1 in adipose tissues causes macrophage recruitment and insulin resistance. J Biol Chem 281:26602-26614

8. Jimenez-Sainz MC, Fast B, Mayor F Jr, Aragay AM (2003) Signaling pathways for monocyte chemoattractant protein 1-mediated extracellular signal-regulated kinase activation. Mol Pharmacol 64:773-782

9. Gordon S, Taylor PR (2005) Monocyte and macrophage heterogeneity. Nat Rev Immunol 5:953-964

10. Mantovani A, Sica A, Sozzani S, Allavena P, Vecchi A, Locati M (2004) The chemokine system in diverse forms of macrophage activation and polarization. Trends Immunol 25:677-686

11. Kanda H, Tateya S, Tamori Y et al (2006) MCP-1 contributes to macrophage infiltration into adipose tissue, insulin resistance, and hepatic steatosis in obesity. J Clin Invest 116:1494-1505

12. Lumeng CN, Bodzin JL, Saltiel AR (2007) Obesity induces a phenotypic switch in adipose tissue macrophage polarization. J Clin Invest 117:175-184

13. Moitra J, Mason MM, Olive M et al (1998) Life without white fat: a transgenic mouse. Genes Dev 12:3168-3181

14. Gavrilova O, Leon LR, Marcus-Samuels B et al (1999) Torpor in mice is induced by both leptin-dependent and -independent mechanisms. Proc Natl Acad Sci U S A 96:14623-14628

15. Kim JK, Gavrilova O, Chen Y, Reitman ML, Shulman GI (2000) Mechanism of insulin resistance in A-ZIP/F-1 fatless mice. J Biol Chem 275:8456-8460

16. Nunez NP, Oh WJ, Rozenberg J et al (2006) Accelerated tumor formation in a fatless mouse with type 2 diabetes and inflammation. Cancer Res 66:5469-5476

17. Nunez NP, Hursting SD, Yakar S, Fowler D, Vinson C (2009) Obesity provides a permissive milieu in inflammation-associated carcinogenesis: analysis of insulin and IGF pathways. Methods Mol Biol 512:29-37

18. Yang SJ, IglayReger HB, Kadouh HC, Bodary PF (2009) Inhibition of the chemokine (C-C motif) ligand 2/chemokine (C-C motif) receptor 2 pathway attenuates hyperglycaemia and inflammation in a mouse model of hepatic steatosis and lipoatrophy. Diabetologia 52:972-981

19. Yamauchi T, Kamon J, Waki H et al (2003) Globular adiponectin protected ob/ob mice from diabetes and ApoE-deficient mice from atherosclerosis. J Biol Chem 278:2461-2468
20. Yamauchi T, Nio Y, Maki T et al (2007) Targeted disruption of AdipoR1 and AdipoR2 causes abrogation of adiponectin binding and metabolic actions. Nat Med 13:332-339

21. Iwabu M, Yamauchi T, Okada-Iwabu M et al (2010) Adiponectin and AdipoR1 regulate PGC-1alpha and mitochondria by $\mathrm{Ca}(2+)$ and AMPK/SIRT1. Nature 464:1313-1319

22. Yamauchi $T$, Tobe $K$, Tamemoto $H$ et al (1996) Insulin signalling and insulin actions in the muscles and livers of insulin-resistant, insulin receptor substrate 1-deficient mice. Mol Cell Biol 16:3074 3084

23. Sartipy P, Loskutoff D (2003) Monocyte chemoattractant protein 1 in obesity and insulin resistance. Proc Natl Acad Sci U S A 100:7265-7270

24. Chen A, Mumick S, Zhang C et al (2005) Diet induction of monocyte chemoattractant protein-1 and its impact on obesity. Obes Res 13:1311-1320

25. Li SL, Reddy MA, Cai Q et al (2006) Enhanced proatherogenic responses in macrophages and vascular smooth muscle cells derived from diabetic $\mathrm{db} / \mathrm{db}$ mice. Diabetes 55:2611-2619

26. Seki E, de Minicis S, Inokuchi S et al (2009) CCR2 promotes hepatic fibrosis in mice. Hepatology 50:185-197

27. Mandrekar P, Ambade A, Lim A, Szabo G, Catalano D (2011) An essential role for monocyte chemoattractant protein-1 in alcoholic liver injury: regulation of proinflammatory cytokines and hepatic steatosis in mice. Hepatology 54:2185-2197

28. Kaneko K, Ueki K, Takahashi N et al (2010) Class IA phosphatidylinositol 3-kinase in pancreatic beta cells controls insulin secretion by multiple mechanisms. Cell Metab 12:619-632

29. Di Gregorio GB, Yao-Borengasser A, Rasouli N et al (2005) Expression of CD68 and macrophage chemoattractant protein-1 genes in human adipose and muscle tissues: association with cytokine expression, insulin resistance, and reduction by pioglitazone. Diabetes 54:2305-2313

30. Chacon MR, Fernandez-Real JM, Richart C et al (2007) Monocyte chemoattractant protein-1 in obesity and type 2 diabetes. Insulin sensitivity study. Obesity (Silver Spring) 15:664-672

31. Dubois PM, Palmer D, Webb ML, Ledbetter JA, Shapiro R (1996) Early signal transduction by the receptor to the chemokine monocyte chemotactic protein-1 in a murine T cell hybrid. J Immunol 156:1356-1361

32. Bost F, Aouadi M, Caron L et al (2005) The extracellular signalregulated kinase isoform ERK1 is specifically required for in vitro and in vivo adipogenesis. Diabetes 54:402-411

33. Hotamisligil GS (2006) Inflammation and metabolic disorders. Nature 444:860-867

34. Werle M, Schmal U, Hanna K, Kreuzer J (2002) MCP-1 induces activation of MAP-kinases ERK, JNK and p38 MAPK in human endothelial cells. Cardiovasc Res 56:284-292

35. Kang K, Reilly SM, Karabacak V et al (2008) Adipocyte-derived Th2 cytokines and myeloid PPARdelta regulate macrophage polarization and insulin sensitivity. Cell Metab 7:485-495

36. Obstfeld AE, Sugaru E, Thearle M et al (2010) C-C chemokine receptor 2 (CCR2) regulates the hepatic recruitment of myeloid cells that promote obesity-induced hepatic steatosis. Diabetes 59:916-925

37. Tamura Y, Sugimoto M, Murayama T et al (2008) Inhibition of CCR2 ameliorates insulin resistance and hepatic steatosis in $\mathrm{db} / \mathrm{db}$ mice. Arterioscler Thromb Vasc Biol 28:2195-2201 